\title{
Sums of Squares, Cubes, and Higher Powers
}

\author{
William C. Jagy and Irving Kaplansky
}

\section{CONTENTS}

\section{Introduction}

2. A Square and Two Cubes, Mixed Style

3. Two Squares and a Cube, Positive Style

4. A Square and Two Cubes, Positive Style

5. Higher Powers

Acknowledgements

References
Any integer is expressible as a sum of two squares and a cube, mixed signs being allowed. We study the analogous question for a square and two cubes, and obtain an affirmative answer in the range from $-4,000,000$ to 2,000,000. For two squares and a cube with everything positive, computations support the possibility that there are only finitely many exceptions. However, $x^{2}+y^{2}+z^{9}$ admits infinitely many positive exceptions.

\section{INTRODUCTION}

The "easier" Waring problems (meaning that the summands are allowed to have mixed signs) have traditionally been attacked, with some success, by locating relevant identities. Recently Elkies and Kaplansky [1995] handled the case of two squares and a cube in this way. It seems reasonable to regard this as the "minimal" case and to propose one square and two cubes as the next step up the ladder. In Section 2 we present what we are able to say about this problem.

In Section 3 we return to two squares and a cube, this time with everything positive. Here it is known that almost all positive integers can be represented. By this we mean that

$$
\lim _{n \rightarrow \infty} \frac{E(n)}{n}=0,
$$

where $E(n)$ is the number of non-represented integers $\leq n$. We shall call such numbers exceptions. Stronger statements are proved in [Roth 1949] and [Halberstam 1950]. The still stronger statement that there are only a finite number of exceptions may be true; we present numerical data.

The final section contains some observations on two squares and an $n$-th power for $n \geq 4$, both in the mixed case and with everything positive. 


\section{A SQUARE AND TWO CUBES, MIXED STYLE}

Here are two identities that do four-ninths of the job:

$$
\begin{aligned}
9 t & =(3 t+3)^{2}+(t-1)^{3}-(t+2)^{3}, \\
3 t+1 & =(3 t+8)^{2}+(t+1)^{3}-(t+4)^{3} .
\end{aligned}
$$

Clearly, the first of these equations shows that any multiple of 9 is a sum of a square and two cubes, and the second does the same for numbers congruent to $1 \bmod 3$.

A search, partly by computer, for identities expressing other linear functions of $t$ in this fashion yielded nothing of interest. However, identities where the left-hand side is a quadratic function in two variables exist in abundance: in $x^{2}+y^{3}+z^{3}$, put $z=u-y$ and treat $u$ as a parameter, getting $x^{2}+3 u y^{2}-3 u^{2} y+u^{3}$.

Some guidance is available in the case of cubes (with mixed signs, of course), which has been thoroughly studied. Every integer not congruent to 4 or $5(\bmod 9)$ is a sum of four cubes, and maybe all are. This is proved by using several identities. See [Mordell 1969, pp. 182-185] and the followup paper [Cohn and Mordell 1972] for the state of the art, including negative results on the prospect for finishing sums of four cubes by identities. The identity

$$
\begin{aligned}
& 6 x^{2}+y^{3}+z^{3}+2 \\
& \quad=(x+1)^{3}-(x-1)^{3}+y^{3}+z^{3},
\end{aligned}
$$

which is exploited in [Davenport and Heilbronn 1937a], gives rise to a similar pessimism concerning $6 x^{2}+y^{3}+z^{3}$. This does not directly jeopardize $x^{2}+y^{3}+z^{3}$, but it leads us to think that the prospect for finishing a square and two cubes by identities is not hopeful.

We therefore sought computational data, and verified that every integer between $-4,000,000$ to $2,000,000$ is expressible as a sum of a square and two cubes. Initially there was a hand computation for the interval from -1000 to 1000 ; this covered all but about 100 of these numbers.
Then two programs were written. The first is, in essence, a program for determining whether a given integer $N$ is a sum of two cubes (mixed signs allowed). Suppose that $N=y^{3}+z^{3}$. Then $N=a b$, where $a=y+z$ and $b=y^{2}-y z+z^{2}$. In $3 y z=a^{2}-b$, set $z=a-y$ to get $3 y(a-y)=a^{2}-b$, or

$$
3 y^{2}-3 a y+a^{2}-b=0 .
$$

A necessary and sufficient condition for this quadratic equation to have rational roots is that the discriminant

$$
(3 a)^{2}-4 \cdot 3\left(a^{2}-b\right)=12 b-3 a^{2}
$$

be a square. Indeed, when $12 b-3 a^{2}$ is a square, $N$ is the sum of two integral (not just rational) cubes; the proof is easy and we leave it to the reader. So the procedure is to factor $N=a b$ in all possible ways and look for a case where $12 b-3 a^{2}$ is a square. In practice the search was speeded up by using information $\bmod 7$ and $\bmod 9$. Then, to hunt for a representation of some $M$ as $x^{2}+y^{3}+z^{3}$, we successively set $x=0,1,2, \ldots$ and applied the test to $N=M-x^{2}$. The question is whether the procedure is going to terminate with success. It always did.

Remark. A pleasant feature of this program is that the number $x$ is the smallest that will do.

The second program adapted ideas sent us by Ford (see acknowledgments at the end of the article). Suitable a priori bounds were chosen for $x,|y|$, and $|z|$. All values of $x^{2}+y^{3}+z^{3}$ that showed up in a specified interval were noted in an array, thus recording for each $m$ in the interval a Boolean value $\chi(m) \in\{0,1\}$, depending on whether the value $m$ ever occurred. Any targets $m$ missed here were then tested by the first program. The largest value of $x$ ever needed occurred in

$$
-1,506,325=105,028^{2}-2186^{3}-837^{3} .
$$

Also noteworthy was

$$
-1227=24122^{2}-872^{3}+433^{3},
$$


the number 24122 being unusually large for such a small target. In addition we did some spot checking, the biggest target being

$$
-500,000,000,001=34514^{2}-7517^{3}-4244^{3} .
$$

\section{TWO SQUARES AND A CUBE, POSITIVE STYLE}

The agenda here is to study the representability of a positive integer in the form $x^{2}+y^{2}+z^{3}$ with $z \geq 0$. We identified 434 exceptions, the largest being $5,042,631$. Ford confirmed this list and extended the search to $50,000,000$; no further exceptions arose.

Congruence properties of these 434 integers are of some interest. We start with the best behavior. (It is to be understood that we are speaking only of this list; we make no conjectures about what happens beyond 50,000,000.) There are no exceptions of the form $8 m+2$ and only one, 813 , of the form $4 m+1$. The 17 exceptions divisible by 4 terminate at 47984 . After that all exceptions have the form $4 m+3$ or $8 m+6$. This behavior was anticipated and is easy to explain. For instance: for numbers of the form $4 m+3$ or $8 m+6$ it is fruitless to try taking the cube to be even. Not foreseen was the fact that the exceptions starting at 137,486 are all of the form $7 m \pm 1$. But this yields to a little Monday morning quarterbacking. Six out of seven cubes are congruent to $\pm 1 \bmod 7$. Thus a number $M$ of the form $7 m \pm 1$ is in extra jeopardy: three times out of seven, $M-z^{3}$ is divisible by 7 , and hence unlikely to be a sum of two squares.

We note at this point that the quadratic form

$$
x^{2}+y^{2}+13 z^{2}
$$

behaves similarly with respect to numbers of the form $8 m+2$ and $4 m+1$; up to $1,000,000$ it represents all numbers of either form with a single exception, 721. This is presumably just a coincidence. From the corollary to Theorem 3 in [Duke and Schulze-Pillot 1990] it is known in this context that there are only a finite number of exceptions.

\section{A SQUARE AND TWO CUBES, POSITIVE STYLE}

It is natural to raise this question too. Nearly sixty years ago Davenport and Heilbronn [1937a] showed that almost all integers are represented. They did this on the way to showing that almost all positive integers are sums of four positive cubes, and they made use of the identity (2.1).

Ford also ran this problem up to $50,000,000$. He found 1,938,144 exceptions, the largest being 49,999,990.

The paper [Ford 1995], among other things, tackles the tougher case of $x^{2}+y^{3}+z^{4}$ and reports on a selective search all the way up to the area of $10^{18}$.

\section{HIGHER POWERS}

Let $n$ be an odd positive integer. Davenport and Heilbronn [1937b] proved that almost all positive integers can be written as $x^{2}+y^{2}+z^{n}$ with $z \geq 0$. Is is possible to strengthen this statement to say that the representation holds from some point on? The following simple result, suggested by exploratory computations, shows that the answer is negative, at least for certain values of $n$ :

Theorem. Let $p$ be a (positive) prime of the form $4 m+1$. Then $216 p^{3}$ cannot be written as $x^{2}+y^{2}+z^{9}$ (even if we are generous and allow $z$ to be negative as well as positive).

Thus the conjecture in [Vaughan 1981, p. 105] is a little too optimistic (it is easy to see that the assumption of no congruence obstructions is satisfied).

Proof. Write $A=216 p^{3}-z^{9}$. Our task is to show that $A$ cannot be a sum of two squares. Assume the contrary. We first find three restrictions on $z$ :

- $z$ cannot be even, for then $\frac{1}{8} A$ is of the form $4 m+3$.

- $z$ cannot be of the form $4 m+1$, for then $A$ is of the form $4 m+3$.

- $z$ cannot be divisible by 3 , for then the exact power of 3 dividing $A$ is 27 . 
Thus $z$ is of the form $4 m+3$ and is prime to 3 . The coup de grâce is delivered by the algebraic factorization

$$
A=\left(6 p-z^{3}\right)\left(36 p^{2}+6 p z^{3}+z^{6}\right) .
$$

The first factor has the form $4 m+3$. It follows that some prime $q$ of the form $4 m+3$ must divide both factors of $A$. Now $q$ cannot be 3 , for 3 does not divide $6 p-z^{3}$. Also, $q$ divides

$$
\left(6 p-z^{3}\right)^{2}-\left(36 p^{2}+6 p z^{3}+z^{6}\right)=-18 p z^{3} .
$$

So $q$ divides $z$. But it also divides $6 p-z^{3}$, and we reach the nonsensical conclusion that $q$ divides $6 p$.

Remarks. (a) See [Vaughan 1981, p. 123, exercises 1 and 2] for a similar result. An interesting contrast to these results is given in exercise 1 on page 35 of the same work.

(b) Many other choices can replace $216 p^{3}$ in the theorem, and 9 can be replaced by any odd composite number (we shall not enter into the details).

(c) In this way, one can show that $x^{2}+y^{2}+z^{n}$, mixed style, cannot represent all integers if $n$ is odd and composite (and in fact there must be an infinite set of positive exceptions and an infinite set of negative exceptions).

(d) On the other hand, if $n$ is an odd prime, it is conceivable that, mixed style, $x^{2}+y^{2}+z^{n}$ represents all integers. Some modest computations for $n=5,7$, and 11 support this possibility.

(e) If $n$ is even and greater than 3 , then $x^{2}+y^{2}-$ $z^{n}$ fails to represent certain sequences of integers obtained in the style of the theorem. In this case, however, there are congruence obstacles as well.

Finally, we report on an investigation of numbers represented by $g=x^{2}+y^{2}+z^{4}$. Here we must of course confine the search to numbers which are sums of three squares. A convenient procedure is to introduce the companion polynomial

$$
h=x^{2}+y^{2}+4 z^{4} .
$$

We see that $g$ represents a number $4 B$ if and only if $h$ represents $B$, and likewise exchanging the roles of $g$ and $h$. At the modest price of working with two polynomials, we can thereby ignore multiples of 4 . Thus in the case of $g$ we stick to numbers that are not divisible by 4 and not congruent to $7 \bmod 8$; for $h$ we stick to numbers congruent to 1 or $2 \bmod$ 4. The largest exceptions we have are $88,540,435$ for $g$ and 345,731,050 for $h$. These exceptions were found by selective fishing in troubled waters; we do not know all smaller exceptions.

\section{ACKNOWLEDGEMENTS}

We are deeply indebted to Kevin Ford, who generously shared with us the results in [Ford 1995], prior to publication. He also sent us the program he used for the computation reported in Section 3. We were able to adapt his ideas to extend our computation in Section 2.

Thanks go also to Leonid Vaserstein. In a letter dated August 3, 1994, he sent us a list of the exceptions up to 4096 for the case of two squares and a positive cube, thereby encouraging us to investigate this further.

\section{REFERENCES}

[Cohn and Mordell 1972] J. H. E. Cohn and L. J. Mordell, "On sums of four cubes of polynomials", J. London Math. Soc. 5 (1972), 74-78.

[Davenport and Heilbronn 1937a] H. Davenport and H. Heilbronn, "On Waring's problem: two cubes and one square", Proc. London Math. Soc. 43 (1937), 73104.

[Davenport and Heilbronn 1937b] H. Davenport and H. Heilbronn, "Note on the additive theory of numbers", Proc. London Math. Soc. 43 (1937), 142-151.

[Duke and Schulze-Pillot 1990] W. Duke and R. Schulze-Pillot, "Representations of integers by positive ternary quadratic forms and equidistribution of lattice points on ellipsoids", Inv. Math. 99 (1990), $49-57$. 
[Elkies and Kaplansky 1995] N. Elkies and I. Kaplansky, Problem 10426, Amer. Math. Monthly 102 (Jan. 1995), 70.

[Ford 1995] Kevin B. Ford, "The representation of integers as sums of unlike powers", J. London Math. Soc. 51 (1995), 14-26.

[Halberstam 1950] H. Halberstam, "Representations of integers as sums of a square, a positive cube, and a fourth power of a prime", J. London Math. Soc., 25 (1950), 158-168.
[Mordell 1969] L. J. Mordell, Diophantine Equations, Academic Press, New York, 1969.

[Roth 1949] K. F. Roth, "Proof that almost all positive integers are sums of a square, a positive cube, and a fourth power", J. London Math. Soc. 24 (1949), $4-13$.

[Vaughan 1981] R. C. Vaughan, The Hardy-Littlewood Method, Cambridge University Press, Cambridge, 1981.

William C. Jagy, Department of Mathematics, Evans Hall, University of California, Berkeley, CA, 94720 (jagy@math.berkeley.edu)

Irving Kaplansky, Mathematical Sciences Research Institute, 1000 Centennial Drive, Berkeley, CA, 94720 (kap@msri.org)

Received December 6, 1994; accepted in revised form June 22, 1995 